\title{
Three new massive companions in the planet-brown dwarf boundary detected with SOPHIE
}

\author{
R.F. Díaz ${ }^{1,2}$, G. Hébrard ${ }^{1,2}$, F. Bouchy ${ }^{1,2}$, I. Boisse ${ }^{1}$, X. Bonfils ${ }^{3,4}$, \\ L. Arnold ${ }^{2}$, X. Delfosse ${ }^{3}$, M. Desort ${ }^{3}$, A. Eggenberger ${ }^{3}$, D. Ehrenreich ${ }^{3}$, \\ T. Forveille ${ }^{3}$, A.-M. Lagrange ${ }^{3}$, C. Lovis ${ }^{4}$, C. Moutou ${ }^{5}$, F. Pepe ${ }^{4}$, \\ C. Perrier ${ }^{3}$, D. Queloz ${ }^{4}$, A. Santerne ${ }^{5}$, N. C. Santos ${ }^{6}$, D. Ségransan ${ }^{4}$, \\ S. Udry ${ }^{4}$, A. Vidal-Madjar ${ }^{1}$ \\ ${ }^{1}$ Institut d'Astrophysique de Paris, CNRS/UPMC, Paris, France [diaz@iap.fr] \\ 2 Observatoire de Haute Provence, 04870 St Michel l'Observatoire, France \\ ${ }^{3}$ Laboratoire d'Astrophysique de Grenoble, Grenoble, France. \\ ${ }^{4}$ Observatoire de Genève, Univ. de Genève, Sauverny, Switzerland. \\ ${ }^{5}$ Laboratoire d'Astrophysique de Marseille, Marseille, France. \\ ${ }^{6}$ Centro de Astrofísica, Universidade do Porto, Porto, Portugal.
}

\begin{abstract}
We report the detection of three new massive companions to mainsequence stars based on precise radial velocities obtained with the SOPHIE spectrograph, as part of an ongoing programme to search for extrasolar planets. The minimum masses of the detected companions range from around 16 Mjup to around 60 Mjup, and therefore lie at both sides of the boundary between massive extrasolar planets and brown dwarves.
\end{abstract}

\section{Introduction}

Only a handful of brown dwarves transiting in front of a stellar primary have been detected so far (Corot-3, Corot-15, NLTT41135, LHS6343C, Wasp-30). These are valuable objects for which the mass and the radius can be -at least in principle- directly measured. On the other hand, the number of objects detected by radial velocity with minimum masses in the brown dwarf regime is only slowly growing. Increasing the statistic of brown dwarfs and massive planets is crucial to understanding planet formation in the high-mass limit.

\section{Observations and Analysis}

We performed radial velocity measurements with the SOPHIE spectrograph, as part of one of the sub-programmes continuously carried out to search for extrasolar planets (Bouchy et al, 2009). SOPHIE is a high-resolution echelle spectrograph fiber-fed from the Cassegrain focus of the 1.93-m telescope at the Haute-Provence Observatory (OHP). It is installed in a thermally-stabilized environment and the dispersive elements are kept at constant pressure in order to provide high-precision radial velocities (Perruchot et al, 2008). Observations were performed in high-resolution mode which produces spectra with resolving power of $\lambda / \Delta \lambda=75000$ at $5500 \AA$.

The spectra were reduced and extracted using the SOPHIE pipeline, and correlated using numerical binary masks corresponding to different spectral types (F0, G2, K5 and M4) to obtain the radial velocities from a gaussian fit to the cross-correlation function.

This is an Open Access article distributed under the terms of the Creative Commons Attribution-Noncommercial License 3.0, which permits unrestricted use, distribution, and reproduction in any noncommercial medium, provided the original work is properly cited. 

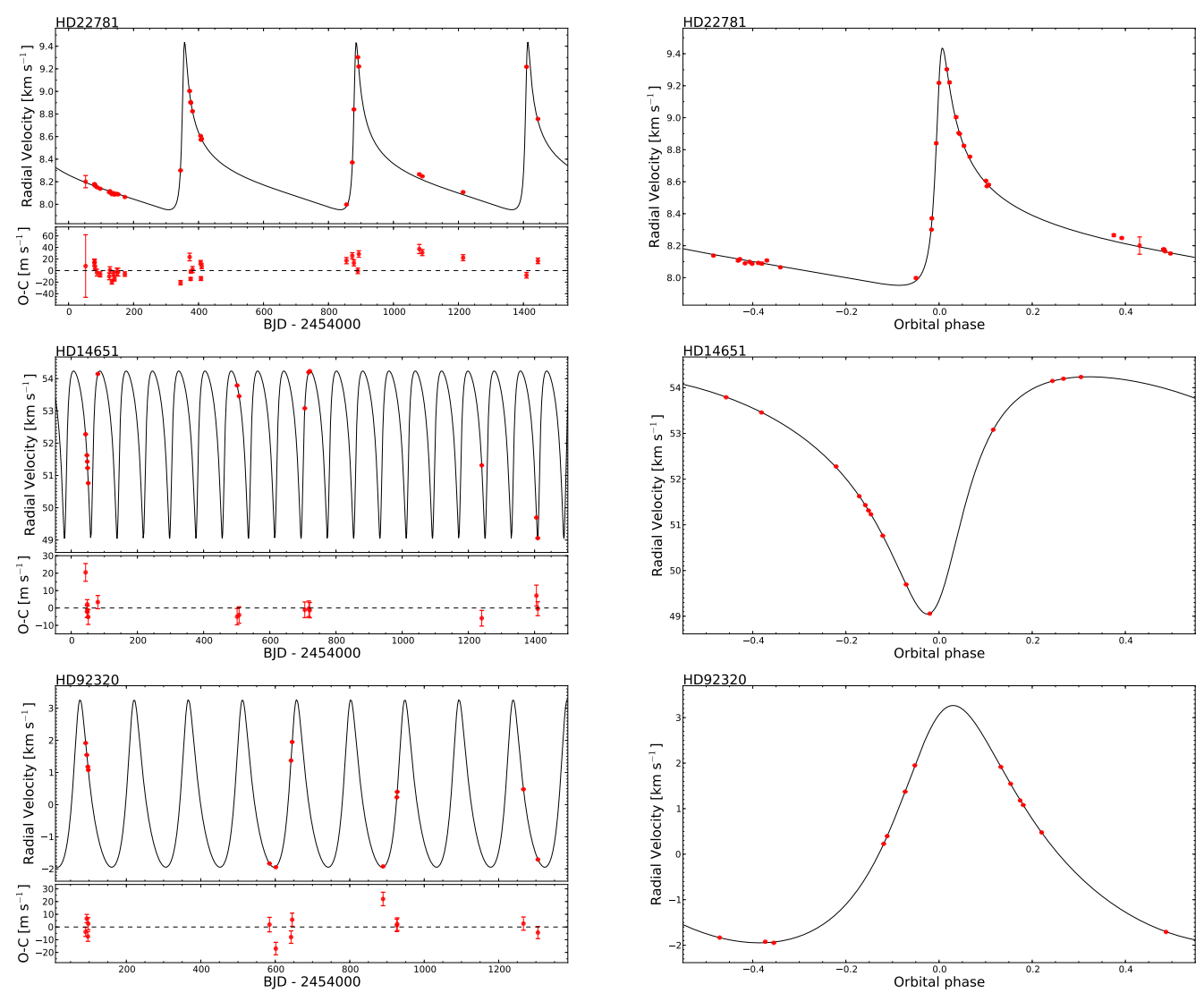

Figure 1: Radial velocity curves for all the three candidates, as a function of time (left column) and as a function of orbital phase (right column). The residuals are also presented in the left column.

The measurements of all these targets exhibit variations greatly in excess of the precision obtained routinely with SOPHIE for these types of stars. Therefore, no periodogram analysis was needed to detect the companion signal. The RVs were fit using a single keplerian orbit model, with none fixed parameters. A genetic algorithm was employed to pick up the starting point for the Levenberg-Marquardt method, which produces the best-fit parameters reported in Table 1 . In Figure 1 we plot the RVs together with the best-fit model and the residuals. We did not detected any significant correlation of the radial velocity with the bisector velocity span, nor any dependence of the amplitude of the velocity variations with the numerical mask used were detected. These facts support the hypothesis that the observed variations are caused by substellar objects orbiting the targets. Additionally, the residuals of HD22781 exhibit a dispersion in excess of the measurement errors, which could be caused by an additional object in the system.

\section{References}

Bouchy F., Hébrard G., Udry S. et al., 2009, A\&A 505, 853

Perruchot S., Kohler D., Bouchy F. et al, 2008, SPIE 7014, 17 
Detection and Dynamics of Transiting Exoplanets

Table 1: Parameters of the host star and the detected companion

\begin{tabular}{|c|c|c|c|c|c|c|c|c|c|}
\hline & \multicolumn{3}{|c|}{ HD22781 } & \multicolumn{3}{|c|}{ HD92320 } & \multicolumn{3}{|c|}{ HD14651 } \\
\hline Sp. Type & \multicolumn{3}{|c|}{ K0V } & \multicolumn{3}{|c|}{ G0V } & \multicolumn{3}{|c|}{ G0V } \\
\hline V & \multicolumn{3}{|c|}{8.78} & \multicolumn{3}{|c|}{8.38} & \multicolumn{3}{|c|}{8.26} \\
\hline$B-V$ & \multicolumn{3}{|c|}{0.85} & \multicolumn{3}{|c|}{0.68} & \multicolumn{3}{|c|}{0.72} \\
\hline $\mathrm{d}[\mathrm{pc}]$ & \multicolumn{3}{|c|}{31.79} & \multicolumn{3}{|c|}{41.48} & \multicolumn{3}{|c|}{39.64} \\
\hline $\mathrm{M}_{*}\left[\mathrm{M}_{\odot}\right]$ & \multicolumn{3}{|c|}{0.78} & \multicolumn{3}{|c|}{1.1} & \multicolumn{3}{|c|}{1.1} \\
\hline $\mathrm{v} \sin i\left[\mathrm{~km} \mathrm{~s}^{-1}\right]$ & \multicolumn{3}{|c|}{1.7} & \multicolumn{3}{|c|}{3.1} & \multicolumn{3}{|c|}{3.0} \\
\hline$<\log \mathrm{R}_{\mathrm{HK}}^{\prime}>$ & \multicolumn{3}{|c|}{-4.8} & \multicolumn{3}{|c|}{-4.7} & \multicolumn{3}{|c|}{-4.3} \\
\hline$\sigma_{\log \mathrm{R}_{\mathrm{HK}}^{\prime}}$ & \multicolumn{3}{|c|}{0.25} & \multicolumn{3}{|c|}{0.06} & \multicolumn{3}{|c|}{0.21} \\
\hline$P[d]$ & 528.10 & \pm & 0.25 & 145.393 & \pm & 0.022 & 79.418 & \pm & 0.002 \\
\hline $\mathrm{T}_{0}$ [BJD-2454000] & 881.51 & \pm & 0.22 & 652.93 & \pm & 0.26 & 458.154 & \pm & 0.051 \\
\hline e & 0.8224 & \pm & 0.0043 & 0.329 & \pm & 0.003 & 0.475 & \pm & 0.001 \\
\hline$\omega[\mathrm{deg}]$ & -42.9 & \pm & 1.0 & -22.53 & \pm & 0.86 & -152.24 & \pm & 0.24 \\
\hline $\mathrm{K}\left[\mathrm{m} \mathrm{s}^{-1}\right]$ & 741.0 & \pm & 14.8 & 2607.0 & \pm & 13.8 & 2595.1 & \pm & 4.1 \\
\hline$a[\mathrm{AU}]$ & 1.18 & \pm & 0.02 & 0.568 & \pm & 0.01 & 0.379 & \pm & 0.006 \\
\hline $\mathrm{M}_{c} \sin i\left[\mathrm{M}_{\mathrm{Jup}}\right]$ & 14.2 & \pm & 0.521 & 67.8 & \pm & 2.4 & 51.5 & \pm & 1.7 \\
\hline
\end{tabular}

